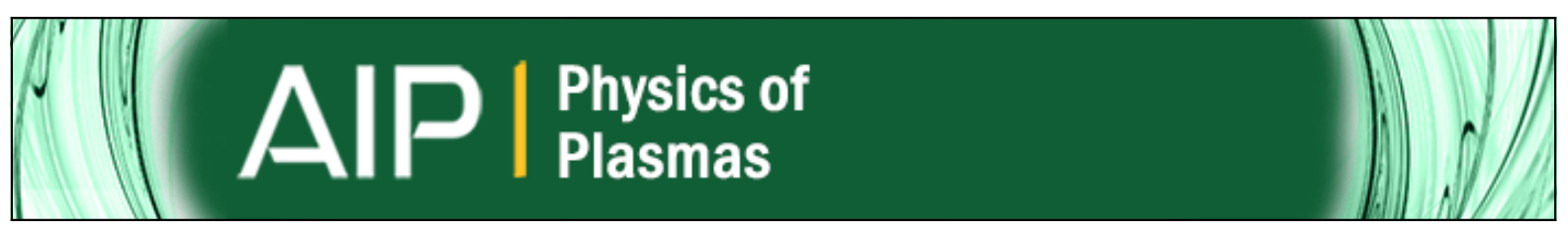

\title{
Model for nonlinear evolution of localized ion ring beam in magnetoplasma
}

W. A. Scales, G. Ganguli, L. Rudakov, and M. Mithaiwala

Citation: Physics of Plasmas (1994-present) 19, 062902 (2012); doi: 10.1063/1.4729330

View online: http://dx.doi.org/10.1063/1.4729330

View Table of Contents: http://scitation.aip.org/content/aip/journal/pop/19/6?ver=pdfcov

Published by the AIP Publishing

AAP Re-register for Table of Content Alerts 


\title{
Model for nonlinear evolution of localized ion ring beam in magnetoplasma
}

\author{
W. A. Scales, ${ }^{1}$ G. Ganguli, ${ }^{2}$ L. Rudakov, ${ }^{3}$ and M. Mithaiwala ${ }^{2}$ \\ ${ }^{1}$ Bradley Department of Electrical and Computer Engineering, Virginia Tech, Blacksburg, \\ Virginia 24061-0111, USA \\ ${ }^{2}$ Plasma Physics Division, Naval Research Laboratory, Washington D.C. 20375, USA \\ ${ }^{3}$ Icarus Research, Inc., P.O. Box 30780, Bethesda, Maryland 20824-0780, USA
}

(Received 6 October 2011; accepted 8 May 2012; published online 15 June 2012)

\begin{abstract}
An electrostatic hybrid model, which investigates the nonlinear evolution of a localized ion ring beam in a magnetoplasma, is described and applied to the generation and evolution of turbulence in the very low frequency (VLF) $\left(\Omega_{c i}<\omega<\Omega_{c e}\right)$ range, where $\Omega_{c i(e)}$ is the ion (electron) gyro frequency. Electrons are treated as a fluid and the ions with the particle-in-cell method. Although the model is electrostatic, it includes the effects of energy loss by convection of electromagnetic VLF waves out of the instability region by utilizing a phenomenological model for effective collisions with the fluid electrons. In comparison with a more conventional electrostatic hybrid model, the new model shows much more efficient extraction of energy from the ion ring beam and reduced background plasma heating over a range of parameters. (C) 2012 American Institute of Physics. [http://dx.doi.org/10.1063/1.4729330]
\end{abstract}

\section{INTRODUCTION}

Beams are commonplace in both space and laboratory plasmas. Electron and ion beams both perpendicular and parallel to ambient magnetic fields can be a source of free energy for a variety of waves. Recently, we have studied the waves that can be generated by a non-relativistic ion ring beam in plasmas $^{1}$ and find that for reasonable beam velocities and densities it can generate waves in the very low frequency (VLF) $\left(\Omega_{c i}<\omega<\Omega_{c e}\right)$ range, where $\Omega_{c i(e)}$ is the ion (electron) gyro frequency. These waves are of particular interest to space plasmas where beams can transport energy and momentum from different parts of the magnetosphere and dissipate them near the Earth thereby affecting the plasma state and, hence, the near-Earth space weather. The VLF saucers ${ }^{2,3}$ observed in the ionosphere are an example of such phenomenon. It is therefore important to understand the extent of the beam energy that is dissipated by the instabilities and how that affects the plasma medium. This is a complex nonlinear problem involving wave-particle interactions and induced scattering of electrostatic waves into electromagnetic waves and vice-e-versa with large disparities in scale sizes that requires three dimensional (3D) electromagnetic particle-incell (PIC) simulations for accurate evaluation. Since such simulations are still impractical, we develop a simpler method to determine the extent of beam energy extraction by the instabilities. Waves in the VLF frequency range have been extensively treated in the literature for various applications, such as plasma heating, ${ }^{4}$ solar applications, ${ }^{5}$ cometary X-rays, ${ }^{6}$ reconnection physics, ${ }^{7}$ and the outer heliosphere. ${ }^{8}$ However, none of these works consider a spatially localized beam in simulations and the effect of induced nonlinear scattering of the quasi-electrostatic lower hybrid waves into whistlers in theoretrical models, which can result in the loss of energy from the region of generation by convection as discussed in our previous work. ${ }^{9}$ The primary focus of this article is to study this important nonlinear effect.
The method we discuss in this article is general but we will choose a specific case to demonstrate its effectiveness. We will consider non-relativistic ion ring beams perpendicular to the ambient magnetic field which can produce quasielectrostatic lower hybrid waves in the VLF frequency range. ${ }^{1}$ Since short wavelength electrostatic waves have a small group velocity they are dissipated in the region where they are generated leading to local plasma heating. However, if these waves are generated with large enough amplitude then induced nonlinear scattering of the lower hybrid waves by thermal electrons or ions can convert them into long wavelength electromagnetic whistler waves. ${ }^{9}$ Electromagnetic waves have large group velocity and propagate out of the region of generation and thereby distribute the beam energy geographically over an extended volume. The question is what fraction of the beam energy can be extracted and radiated away from the region of generation by this process?

Quasi-linear evolution of the lower-hybrid waves indicates that initially the waves grow and saturate at the expense of the beam energy, but subsequently the wave energy returns to the ring beam leading to thermalization of the ring ions. ${ }^{10-12}$ The quasi-linear picture is an incomplete representation of the realistic dynamics of a system. Nonlinear processes can affect the saturation level and hence the magnitude of the net beam energy extraction. ${ }^{13}$ In general, the energy extracted is shared between beam thermalization, wave generation, and plasma heating due to quasi-linear and nonlinear processes. A 2D electromagnetic PIC simulation of an ion ring beam reported a $20 \%$ extraction of the initial beam energy. ${ }^{14}$ McClements et al. ${ }^{14}$ used a periodic system in which the beam is not localized in space. The energy extracted from the ring beam remains within the region of wave generation but is expended into nonlinear processes and the lower hybrid waves saturate at low amplitudes. The simulation showed that if there are nonlinear processes for the energy to flow into, then only a fraction of it returns to the ring ions and, consequently, a larger fraction of beam 
energy may be extracted as compared to the quasi-linear picture which finally appears as ion and electron heating. Since the energy extraction occurs initially by electrostatic waves, we may circumvent a time intensive $3 \mathrm{D}$ electromagnetic PIC simulation of the process by using a simpler electrostatic hybrid model for estimating the energy extracted. However, the electrostatic model must include the effects of the essential nonlinear processes by which the energy is expended through a realistic parameterization. This may lead to economies in CPU time and memory, making the simulations more practical. In the following paragraphs, we discuss the construction of such a model.

\section{A. Parameterization of nonlinear processes}

The model of McClements et al. is periodic and hence it represents an infinite system. In this situation, the rate of wave energy convection out of the region of creation, $V_{g} / L \rightarrow 0$ since $L \rightarrow \infty$, where $V_{g}$ and $L$ are group velocity of the waves and the spatial extent of the beam, respectively. Consequently, the energy extracted from the beam remains within the system but is redistributed into plasma heating and nonlinear processes. In reality, however, the beams are localized in a finite extent of space. Although the energy may be extracted from the beam locally through the generation of electrostatic waves, they may be nonlinearly scattered into electromagnetic waves which have large $V_{g}$ and can propagate out of the region of generation. ${ }^{9}$ In addition, for the VLF turbulence, the dominant nonlinear (vector nonlinearity) terms in plasma equation of motion are three dimensional. The essential 3D effects may be simulated in a $2 \mathrm{D}$ simulation by placing the external magnetic field at an angle out of the simulation plane. ${ }^{9}$ It is therefore important to incorporate these features in simpler simulation models investigating the beam energy extraction phenomenon. The simpler parameterized model that we envision is based on the following observations of the phenomenon:

1. The beam is localized in space and hence excites quasielectrostatic lower hybrid waves in this "source" region.

2. For low $\beta$ near-Earth plasmas, the rate of nonlinear induced scattering (i.e., nonlinear Landau damping) of the lower hybrid waves can be large and, consequently, they are rapidly scattered into long wavelength electromagnetic whistlers/magnetosonic waves avoiding linear damping.

3. The group velocity of the electromagnetic waves is large and their rate of convection exceeds local damping rates and they instantly propagate out of the region of their generation.

4. Because of rapid whistler/magnetosonic wave convection, the energy extracted from the beam is instantly removed from the region of localization of the beam.

The first point above allows us to use an electrostatic simulation model. The second and third points allow us to use a hybrid model with electrons as a fluid because we need not explicitly include the process of nonlinear scattering, but rather parameterize this process with an effective collision fre- quency. The fourth point allows us to utilize a local simulation of the source region with an effective parameterization of energy sink which can instantly remove the energy from the system as it is extracted from the beam to represent the wave convection out of the region of generation. These simplifications make the simulation of this complex nonlinear process tractable, practical, and CPU time friendly.

\section{MODEL}

A two dimensional 2D periodic electrostatic hybrid simulation model has been developed to investigate the phenomenology just described. Three plasma species are considered. The two ion species are treated with the PIC method in which background Maxwellian velocity distribution ions have density $n_{b i}$ and velocity ring distribution ions, which provide free energy for lower hybrid wave generation, have density $n_{r i}$. The electrons are modeled as a fluid. To determine the electrostatic potential $\varphi$, the condition for current $\vec{J}$ continuity is utilized

$$
\nabla \bullet \vec{J}+\frac{\partial \rho}{\partial t}=0 \quad \text { or } \quad \nabla \bullet n_{e} \vec{v}_{e}+\frac{\varepsilon_{0}}{e} \frac{\partial \vec{E}}{\partial t}=\nabla \bullet \Gamma_{i}
$$

where $n_{e}$ and $\vec{v}_{e}$ are the electron density and velocity, respectively, $e$ and $\varepsilon_{0}$ are the unit charge and vacuum permittivity, respectively, and $\Gamma_{i}$ is the total ion particle flux. The electrostatic field is determined from $\vec{E}=-\nabla \varphi$. It is assumed that the simulation is in the $x-y$ plane and the magnetic field $\vec{B}$ is in the $x-z$ plane at an angle $\theta_{B}$ to the simulation plane. The magnetic field unit vector is given by $\hat{b}=\cos \theta_{B} \hat{x}+\sin \theta_{B} \hat{z}$. It should be noted that the ion particles have velocities in three dimensions while their positions are calculated only in the two-dimensional simulation plane. Quasi-neutrality is used to determine the electron density $n_{e}=n_{i}=n_{b i}+n_{r i}$. The electron velocity is decomposed into components perpendicular $v_{e \perp}$ and parallel $v_{e \|}$ to the magnetic field. Nominally, the electron velocity perpendicular to the magnetic field can be written including both linear and nonlinear inertial terms in this case as

$$
\vec{v}_{e \perp}=\frac{\vec{E} \times \hat{b}}{B}-\frac{1}{\Omega_{c e} B}\left(\frac{\partial \vec{E}_{\perp}}{\partial t}+v_{e \|} \cos \theta_{B} \hat{x} \bullet \nabla \vec{E}_{\perp}\right)
$$

where $\Omega_{c e}$ and $B$ are the electron cyclotron frequency and background magnetic field, respectively. A parallel electron continuity equation is used for calculating the electron velocity along the magnetic field $v_{e} \|$ where $v_{t e}$ is the electron thermal velocity. It is given by

$$
\begin{aligned}
\frac{\partial v_{e \|}}{\partial t}= & -\frac{1}{B}(\hat{b} \times \nabla \varphi) \bullet \nabla v_{e \|}-\cos \theta_{B} v_{e \|} \frac{\partial v_{e \|}}{\partial x} \\
& -\cos \theta_{B} v_{t e}^{2} \frac{1}{n_{i}} \frac{\partial n_{i}}{\partial x}-\cos \theta_{B} \frac{q_{e}}{m_{e}} \frac{\partial \varphi}{\partial x} .
\end{aligned}
$$

An equation for calculating the electrostatic potential can be obtained by combining Eqs. (1) and (2) which is given by 


$$
\begin{aligned}
\nabla \bullet\left(n_{i}+\frac{\Omega_{c e}^{2}}{\omega_{p e}^{2}} n_{e 0}\right) \nabla_{\perp} \frac{\partial \varphi}{\partial t}= & -\Omega_{c e} \nabla \bullet n_{i}(\hat{b} \times \nabla \varphi) \\
& -\cos \theta_{B} \nabla \bullet n_{i} v_{e} \| \frac{\partial}{\partial x} \nabla_{\perp} \varphi \\
& -\cos \theta_{B} \Omega_{c e} B \frac{\partial}{\partial x} n_{i} v_{e} \| \\
& +\Omega_{c e} B \nabla \bullet \Gamma_{i},
\end{aligned}
$$

where $n_{e 0}$ and $\omega_{p e}$ are the initial electron density (equal to the initial ion density) and plasma frequency, respectively, and $\nabla_{\perp}=\hat{x} \sin ^{2} \theta_{B} \partial / \partial x+\hat{y} \partial / \partial y$. This expression coupled with Eq. (3) forms a complete set of equations along with the standard PIC advance for the ion equations of motion.

A key goal of our phenomenological model, however, is to include an effective parameterization of the energy sink due to the nonlinear scattering of the ring beam generated lower hybrid waves into electromagnetic VLF (whistler) waves and their convection out of source region. We will account for this process as wave energy loss through effective electron-ion collisions. It is assumed that there is some mechanism such as thermal conduction along the magnetic field lines, which does not allow the electron temperature to change. In this case, the fluid electron velocity (2) may be written in a simple form that includes effective collisions,

$$
\vec{v}_{e \perp}=\frac{\vec{E} \times \hat{b}}{B}-\frac{\nu_{e f f}}{\Omega_{c e}} \frac{\vec{E}_{\perp}}{B}-\frac{1}{\Omega_{c e} B} \frac{\partial \vec{E}_{\perp}}{\partial t},
$$

where $\nu_{\text {eff }}$ is a time-varying anomalous electron collision frequency which depends on the wave amplitude. The effective collisions are modeled as

$$
\nu_{e f f}(t)=\delta \gamma\left(\exp \left[\frac{\varepsilon(t)}{\varepsilon_{m}}\right]-1\right),
$$

where $\gamma, \varepsilon$, and $\varepsilon_{m}$ are the linear growth rate of the lower hybrid waves due to the ring plasma, the time dependent electrostatic field energy $\left(\varepsilon(t) \sim|\vec{E}(t)|^{2}\right)$, and the maximum value of the field energy from a benchmark simulation, respectively. $\delta$ is a scaling constant that is varied to study the impact of the strength of the effective collisions. The parallel electron momentum equation in this case can be written as

$$
\begin{aligned}
\frac{\partial v_{e \|}}{\partial t}= & -\cos \theta_{B} \nu_{e f f} v_{e \|}-\cos \theta_{B} v_{e \|} \frac{\partial v_{e \|}}{\partial x}-\cos \theta_{B} v_{t e}^{2} \frac{1}{n_{i}} \frac{\partial n_{i}}{\partial x} \\
& -\cos \theta_{B} \frac{q_{e}}{m_{e}} \frac{\partial \varphi}{\partial x} .
\end{aligned}
$$

Equation (4) for calculation of the electrostatic potential can be rewritten as

$$
\begin{aligned}
\nabla \bullet & \left(n_{i}+\frac{\Omega_{c e}^{2}}{\omega_{p e}^{2}} n_{e 0}\right) \nabla_{\perp} \frac{\partial \varphi}{\partial t} \\
= & -\nu_{e f f} \nabla \bullet n_{i} \nabla_{\perp} \varphi-\Omega_{c e} \nabla \bullet n_{i}(\hat{b} \times \nabla \varphi) \\
& -\cos \theta_{B} \nabla \bullet n_{i} v_{e}\left\|\frac{\partial}{\partial x} \nabla_{\perp} \varphi-\cos \theta_{B} \Omega_{c e} B \frac{\partial}{\partial x} n_{i} v_{e}\right\| \\
& +\Omega_{c e} B \nabla \bullet \Gamma_{i} .
\end{aligned}
$$

Equations (6)-(8) along with the PIC equations for the ions constitute the phenomenological model that will be used for the following investigation. In these equations, the terms proportional to $\nu_{\text {eff }}$ parameterize the effects of nonlinear scattering. When $\theta_{B}=\pi / 2$, the effects of the vector nonlinearity are isolated. Equations (7) and (8) are coupled and solved with pseudo-spectral methods using a second-order Runge-Kutta time advance. The potential Equation (8) for each Runge-Kutta step is nonlinear in the Fourier spectral domain and is solved with an iterative procedure.

\section{RESULTS}

The model just described has been utilized to investigate nonlinear evolution during ring beam plasma interactions. Although the model described is general, the parameter regime for the application considered here is for a space plasma reasonably representative of the Earth's ionosphere where oxygen ions dominate. The ratio of $\omega_{p e} / \Omega_{c e}=4$ or 5 and $T_{e} / T_{i}=1$. The background ion mass to electron ratio was taken to be $m_{b i} / m_{e}=10^{4}$ which is an approximation of the oxygen to electron mass ratio in the Earth's ionosphere. Typically, $\beta_{e}=\left(\omega_{p e} v_{t e} / \Omega_{c e} c\right)^{2} \sim 10^{-4}$ for altitudes of interest. The ratio of the ring plasma to background ion density, $n_{r i} / n_{b i}$, is varied from 0.25 to 1.0 to access the results and 49 simulation particles per cell are used in all cases for the background ion density. The cold ring plasma speed is $v_{r}$ $=10 v_{t i}$ where the background ion thermal velocity is $v_{t i}$. In the simulation, $v_{r} \sim 10 \mathrm{~km} / \mathrm{s}$ whereas the Alfven speed is $\sim 1000 \mathrm{~km} / \mathrm{s}$. Thus $v_{r}$ is subcritical to the generation of Alfven waves and the electromagnetic effects discussed by Omel'chenko et al. ${ }^{15}$ are negligible. Such a velocity is representative of a flux of ionized barium atoms released from a sounding rocket using shaped charge to accelerate them ${ }^{16}$ or released from spacecraft in orbit. ${ }^{17}$ The ion and ring mass ratio is varied with values $m_{r i} / m_{b i}=1,3$, and 9 to access the effects. The simulation box size for all results is 128 by 128 grid cells with grid cell size approximately equal to the Debye length $\lambda_{D}$ which is sufficient to resolve the most unstable linear mode while conserving computational time. Several simulations with a larger box size (256 by 256) were performed which show qualitatively similar results and will be commented on where appropriate. In the case of this study, the maximum growth rate is roughly approximated from linear theory ${ }^{1}$ by $\gamma_{\max } \approx 0.3 \omega_{l h}\left(n_{r i} m_{b i} / n_{b i} m_{r i}\right)^{2 / 5}$ at the wavenumber of maximum growth $k_{\max } \approx \omega_{l h} / v_{r}$, where $\omega_{l h}$ is the lower hybrid frequency.

\section{A. Comparison with linear electron dynamics model}

For simulating lower-hybrid turbulence it is assumed that the magnetic field is in the $x-z$ plane at an angle $\theta_{B}=\pi / 2$. Though it is permissible to consider the case when the magnetic field is at an arbitrary angle in $x-z$ plane, this would lead to other (scalar) nonlinear effects due to parallel electron dynamics of Eq. (7) in addition to effective collisions $v_{\text {eff }}$. This will be studied in the future. However, as discussed in Ganguli et al., ${ }^{9}$ these scalar nonlinearities are much slower than the dominating vector nonlinearity considered here. It is therefore instructive to consider a comparison of the effective 
collision model that results with a standard electrostatic hybrid simulation model utilizing linear electron dynamics for ion waves corresponding to the case $\theta_{B}=\pi / 2$. The first order (linear) electron $n_{e 1}$ and potential perturbations $\varphi_{1}$ are related by the expression

$$
n_{e 1}=\frac{\varepsilon_{0}}{e} \frac{\omega_{p e}^{2}}{\Omega_{c e}^{2}} k^{2} \varphi_{1}
$$

in the spectral domain where $k$ is the wavenumber. This implies Poisson's equation may then be used to determine the electrostatic potential as

$$
\nabla^{2} \varphi=\frac{e}{\varepsilon_{0}} \frac{1}{\left(1+\omega_{p e}^{2} / \Omega_{c e}^{2}\right)}\left(n_{0}-n_{i}\right),
$$

where $n_{0}$ is the initial background plasma density. The background total PIC ion density is denoted by $n_{i}$.

The linear electron model gives similar results to the conventional hybrid model when $\theta_{B}=\pi / 2$ and $\nu_{\text {eff }} \rightarrow 0$. Fig. 1 shows a benchmark comparison of the two models for a small value of $\delta=5 \times 10^{-4}$ and also $\varepsilon_{m} / K E^{r i}(t=0)$ $=4 \times 10^{-4}$ where $K E^{r i}(t=0)$ is the initial ring kinetic energy. Again, the linear model has the potential solved with Eq. (10) and the effective collision model has the potential solved with Eq. (8). The case shown considers $n_{r i} / n_{b i}$ $=0.25$ and $m_{b i} / m_{r i}=1$ which will also be considered in more detail for the case when effective collisions are important. It is observed that the electrostatic field energy $W_{E}$ (normalized to $K E^{r i}(t=0)$ ) in the linear growth phase is very similar for both models and nonlinear saturation occurs near time $\omega_{l h} t \approx 50$. The background plasma electrostatic field energy fluctuation level for the linear and effective collision models are of order $1 \times 10^{-6}$ and $1 \times 10^{-7}$, respectively, which shows there is wave growth for over three to four orders of magnitude for these parameters. The growth

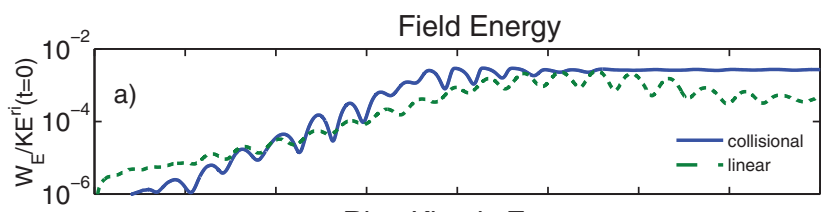

Ring Kinetic Energy
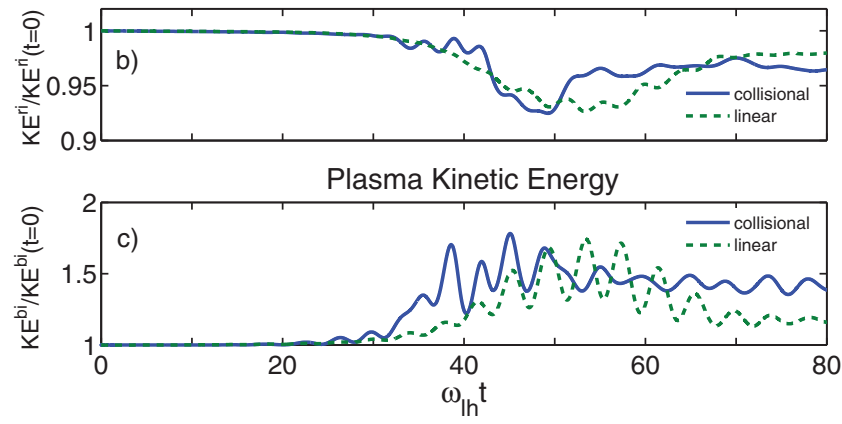

FIG. 1. Comparison of (a) electrostatic field energy, (b) ring kinetic energy, and (c) plasma ion kinetic energy, for linear electron and effective collisional electron models for $n_{r i} / n_{b i}=0.25$ and $m_{b i}=m_{r i}=1$. Note $\delta=5 \times 10^{-4}$ indicating negligible impact of effective collisions. rate for the fastest growing mode from linear theory ${ }^{1}$ is $\gamma_{\max } / \omega_{l h} \approx 0.16$ and the growth rate from the simulation field energy, which contains all modes, is estimated to be $\gamma / \omega_{l h} \approx 0.12$ and 0.10 from the linear and collisional electron models, respectively, which are in reasonable agreement. Simulations with a larger computational domain show slightly better agreement (not shown). The wavenumber of maximum growth during the linear growth phase is as predicted by linear theory with $k_{\max } \approx \omega_{l h} / v_{r}$. Figures $1(\mathrm{~b})$ and $1(\mathrm{c})$ show the ring and plasma kinetic energies, $K E^{r i}$ and $K E^{b i}$, respectively, normalized to their initial values. Figure 1(b) shows there is initially a release of energy from the ring ions prior to saturation of the field energy with both models and then some of the energy returns to the ring ions to thermalize them resulting in increase in the net ring energy from its minimum as described earlier. ${ }^{10}$ This ultimately results in release of no more than a few percent of the ring energy in both models. There is also comparable heating associated with the background ions in both models that maximize around the time of saturation of the electrostatic energy.

Figure 2 shows model comparison results for the same plasma parameters as in Figure 1, i.e., $n_{r i} / n_{b i}=0.25$ and $m_{b i} / m_{r i}=1$. However, in this case, in Eq. (6) $\delta=0.1$ which indicates that the impact of the effective collisions is important. This circumstance will be the focus of all subsequent results to be discussed. The simulations in the present case are run to time $\omega_{l h} t=400$. It is observed that the electrostatic field energy $W_{E}$ (now normalized to the parameter $\varepsilon_{m}$ ) in the linear growth phase is very similar again for both models and nonlinear saturation occurs near time $\omega_{l h} t \approx 50$. The saturation amplitude for collisional electrons occurs when the ratio $W_{E} / \varepsilon_{m} \approx 1$. Figure 2 (b) shows there is initially a release of energy from the ring ions prior to saturation of the field energy with both models as before; however, after saturation of the electrostatic field energy, there is continued release of energy in the effective collision model resulting in
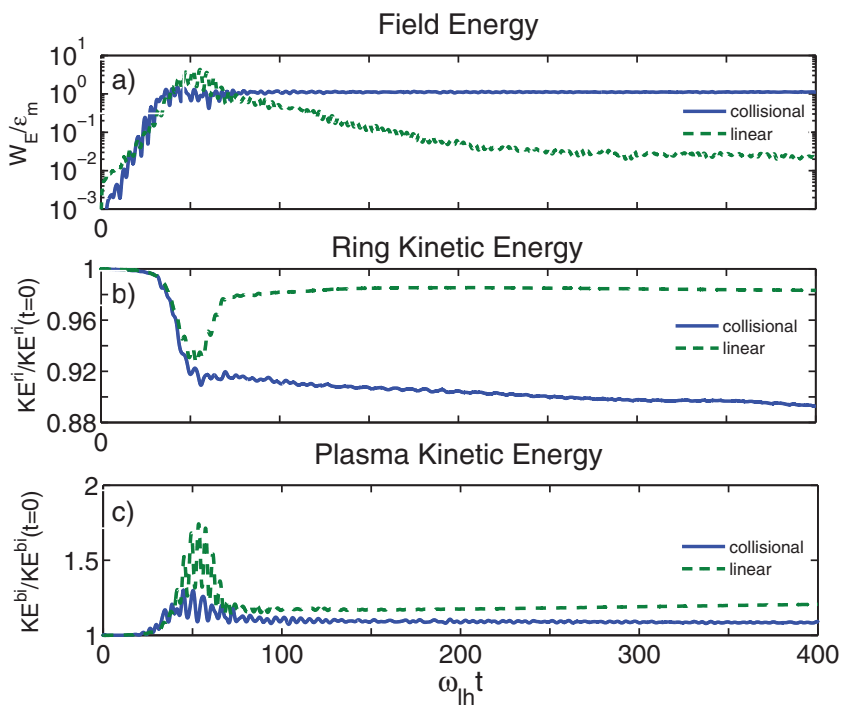

FIG. 2. Comparison of (a) electrostatic field energy, (b) ring kinetic energy, and (c) plasma ion kinetic energy, for linear electron and effective collisional electron models for $n_{r i} / n_{b i}=0.25$ and $m_{b i}=m_{r i}=1$ with $\delta=10^{-1}$. 
substantially more release in ring energy by the end of the simulation ( $11 \%$ compared to $2 \%$ ). There is no decay of field energy in the effective collision model and there is continued slow extraction of kinetic energy from the ring. Figure 2(c) shows energy of the background ions which at the end of simulation shows reduced heating of the effective collision model relative to the linear model.

\section{B. Mass and density dependence}

Results for increased ring mass are shown in Figure 3 with ratio $m_{r i} / m_{b i}=9$. The simulations are now run to time $\omega_{l h} t=1000$, since the growth rate is slower for the larger ring mass. ${ }^{1}$ Again comparisons between the linear electron response model of Eqs. (9) and (10) and the effective collision model are shown. The behavior for the ring and background kinetic energy is shown. There is substantially more extraction of energy from the ring when effective collisions to model VLF wave convection is included in the higher mass case in Figure 3(a). It can be noted in general the release of energy is slower in the high mass case relative to Figure 2(b). The increased ring mass produces significantly larger background plasma (ion) heating in the linear electron response model in comparison to Figure 2(c). When effective collisions are included, the background plasma heating is negligible as observed in Figure 3(b).

It is instructive to consider the velocity distribution functions of the ring $f\left(v_{\perp}\right)$ and plasma $f\left(v_{x}\right)$ ions. Figure 4 shows a comparison of the distributions functions for the linear electron response and effective collision models for $n_{r i} / n_{b i}=0.25$ and $m_{r i} / m_{b i}=1$ and 9 . The distribution functions are shown at the end of the simulation for both models. For the small ring mass case, in the linear electron response model, it is observed that an energetic tail develops on the background plasma distribution due to the development of the lower hybrid instability and their Landau damping. There is also significant thermalization of the cold ring distribution function. Incorporating effective electron collisions greatly suppresses the energetic tail on the background ion distribution function. There is less thermalization of the ring plasma and greater reduction of the ring speed. The trend is quite
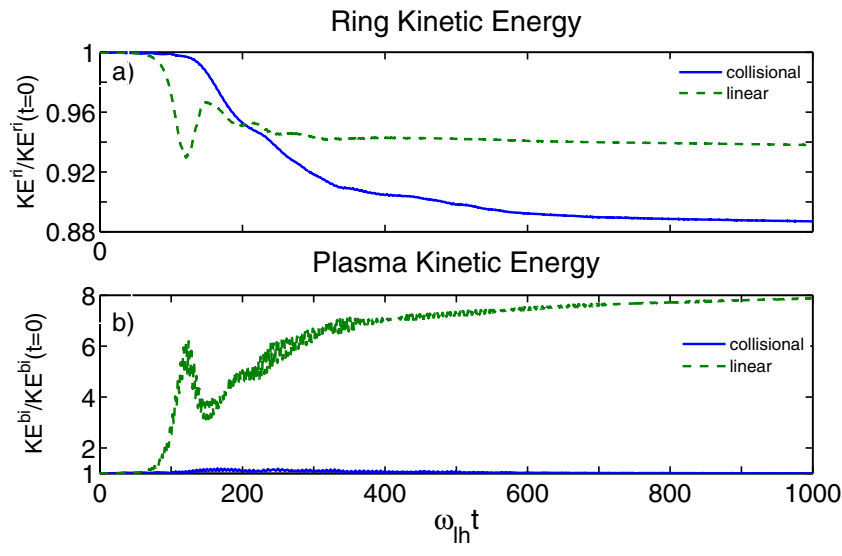

FIG. 3. Comparison of (a) ring kinetic energy and (b) plasma kinetic energy for linear electron and effective collisional electron models for $n_{r i} / n_{b i}=0.25$ and $m_{r i} / m_{b i}=9$ with $\delta=10^{-1}$.
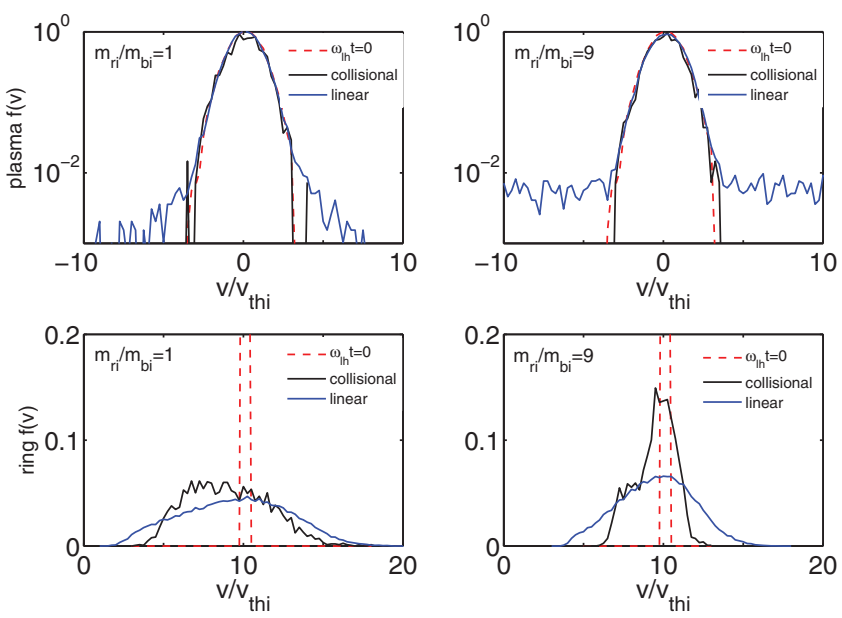

FIG. 4. Comparison of velocity distribution functions of linear electron response and effective collision models for varying ring mass with $\delta=10^{-1}$. Note in both cases, effective collisions suppress energetic tail on background ions as well as thermalization on ring ions. More effective slowing down of ring ions is also produced.

similar for the higher ring mass case. There is a much higher energy tail on the background ion in the linear electron model, but it is essentially eliminated in the effective collision model. However, the effective collisions reduce the thermalization and allow for greater reduction of the ring ion speed, which ultimately leads to more efficient extraction of ring energy.

Increasing the ring density to $n_{r i} / n_{b i}=1$ shows similar trends as the lower density case. The effective collisions shows increased efficiency of extraction of energy from the ring relative to the linear electron response model; however, this increase is less as the ring mass is increased. Figure 5 shows the ring energy for cases $m_{r i} / m_{b i}=1$ and 9 . It is observed that there is substantial increase in extraction efficiency for the lower mass ratio case. For the higher mass ratio case, there is a relatively small difference in the efficiency of extraction between the two models although the effective collision model is still superior. The background ion distribution for this case shows strong core, as opposed to tail, heating, typical of strong turbulence. In this case, since a larger amount of energy is extracted it leads to strong turbulence. Finally, it is important to note for such high ring density and mass the linear electron response model assumption is most likely beginning to break down and care must be taken in considering the validity of the comparison.

\section{DISCUSSION}

In the 2D quasilinear theory of the ring-beam distribution, lower-hybrid waves initially grow and saturate at the expense of the beam energy, and subsequently, the ring ions diffuse so as to limit the growth of wave energy. This is demonstrated in the simulation results of Figure 2 for the linear model (green dotted lines). The ring distribution releases its energy to the waves, the waves grow and saturate, while the ring ions diffuse and ultimately the wave energy is returned to the ring. Though Figure 2(c) (linear model) shows that some energy has gone to plasma kinetic energy (ion heating). 

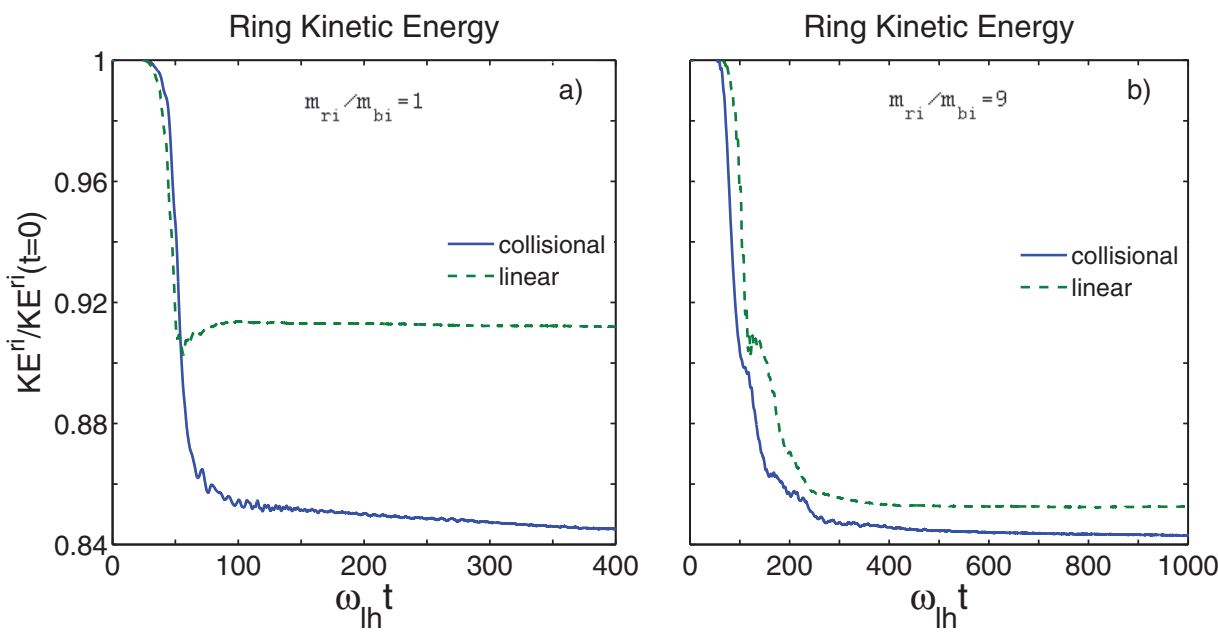

FIG. 5. Ring kinetic energy comparison for linear electron response and effective collisional model for $n_{r i} / n_{b i}=1$ and (a) $m_{r i} / m_{b i}$ $=1$ and (b) $m_{r i} / m_{b i}=9$ with $\delta=10^{-1}$.
However, nonlinear processes can affect the saturation level and, hence, the magnitude of the net beam energy extraction. Nonlinear scattering will pump energy from wavenumbers $k_{1}$ to $k$, where waves with energy density $W_{k}$ are not resonant with the ring particles, and will not be absorbed according to the $2 \mathrm{D}$ quasilinear description. ${ }^{13}$ Thus the wave energy $W_{k 1}$ will be determined by

$$
\frac{\partial W_{k 1}}{\partial t}=2\left(\gamma_{k 1}^{L}-\gamma_{k 1}^{N L}\left(W_{k}\right)\right) W_{k 1}
$$

The nonlinear growth rate $\gamma_{k 1}^{N L}\left(W_{k}\right)$ depends on the amplitude of waves $W_{k}$. The amplitude $W_{k}$ is similarly determined by the scattering rate $\gamma_{k}^{N L}\left(W_{k 1}\right)$ of electrostatic lower hybrid waves to electromagnetic whistlers and their loss due to convection away from the ring region ${ }^{9}$

$$
\frac{\partial W_{k}}{\partial t}=2\left(\gamma_{k}^{N L}\left(W_{k 1}\right)-\gamma_{\text {loss }}\right) W_{k} .
$$

In the 2D electrostatic hybrid simulation model presented here, the loss rate $\gamma_{\text {loss }}$ is represented by $\nu_{\text {eff }}(6)$, and the maximum wave energy density $W_{k 1}$ is set by $\varepsilon_{m}$. Equation (12) shows that at steady state the rate of energy loss is equal to the nonlinear scattering rate, thus $\nu_{\text {eff }}$ is equivalent to $\gamma^{N L}$ in our simulations. For the simulations in Figures 2 and 3, the maximum wave energy $\varepsilon_{m}$ is set equal. In the absence of the effective collisions representing convection of energy, the wave amplitude is much larger since there is nine times more energy in the ring distribution in the simulation presented in Figure 3 compared to that in Fig. 2. When the effects of nonlinear scattering is accounted for by $\nu_{\text {eff }}$ the waves in both cases saturate at the same low amplitude because $\nu_{\text {eff }}$ depends strongly on $\varepsilon_{m}$ which is kept unchanged in the two cases.

Nonlinear effects also affect the time scale of relaxation. Since all the energy extracted from the ring distribution does not return to the ring ions, the time scale for quasilinear relaxation of the ring will be slower ${ }^{13}$ and can be estimated as $1 / \tau_{N L} \sim \gamma^{L} \varepsilon_{m} / E_{\text {ring }}$, where $E_{\text {ring }}=m_{r i} v_{r}^{2} / 2$ is the initial ring energy. Thus, the timescale for relaxation is $\left(m_{r i} / m_{b i}\right)^{3 / 5}$ times longer for the simulation of Figure 3 than Figure 2. Indeed, the simulation results show that it takes over twice as long for the ring distribution to release $10 \%$ of its initial energy in Figure 3 than Figure 2.

\section{CONCLUSIONS}

A new electrostatic hybrid model has been presented for investigating a localized ion ring beam interaction with a magnetoplasma producing waves in the lower hybrid frequency range. The object was to include effects of convection of VLF waves out of the localized region in a computationally efficient manner. It was demonstrated that the phenomenological collisions on the electron fluid appear to successfully allow this important electromagnetic process into the electrostatic model. This VLF wave convection is critical since it is clear that it has fundamental effects on the nonlinear evolution. For a range of parameters of the ring beam relative to the background plasma density $n_{r i} / n_{b i} \leq 1$ and ring mass relative to background plasma mass $1 \leq m_{r i} / m_{b i} \leq 9$, there is a significant increase in the efficiency in extraction of energy from the ring into generation of waves over that predicted by a pure electrostatic model. This is accompanied with a reduced heating of the background plasma and formation of high energy tails on the ion distribution functions for a ring velocity in the range for a typical space experiment, $v_{r}=10 v_{t i}$. Though we have studied lower-hybrid turbulence assuming that the magnetic field is in the $x-z$ plane at an angle $\theta_{B}=\pi / 2$, our simulation model, Eqs. (5)-(8), allows us to study arbitrary angles. If nonlinear effects due to parallel electron dynamics of Eq. (7) are included, it leads to an additional limitation of the wave amplitude and also enhances the efficiency of energy extraction from the ring. This will be presented in a future article. The methodology here is general and can be applied to other applications. The results provide important insight to the design of future space experiments which may utilize strategies involving the release of neutral atoms in space plasmas to generate VLF waves.

\section{ACKNOWLEDGMENTS}

The work at Virginia Tech was supported by the Office of Naval Research MURI program. This work at NRL was 
supported by Naval Research Laboratory Base Program. The authors are grateful to $\mathrm{H}$. Fu for useful discussions and help.

${ }^{1}$ M. Mithaiwala, L. Rudakov, and G. Ganguli, Phys. Plasmas 17, 042113 (2010).

${ }^{2}$ H. G. James, J. Geophys Res. 81, 501, doi: 10.1029/JA081i004p00501 (1976).

${ }^{3}$ R. E. Ergun, C. W. Carlson, J. P. McFadden, R. J. Strangeway, M. V. Goldman, and D. L. Newman, Geophys Res Lett. 28, 3805, doi: 10.1029/ 2001GL013024 (2001).

${ }^{4}$ J. B. McBride, E. Ott, J. P. Boris, and J. H. Orens, Phys. Plasmas 15, 2367 (1972).

${ }^{5}$ R. Bingham, J. M. Dawson, and V. D. Shapiro, J. Plasma Phys. 68, 161-172 (2002).

${ }^{6}$ V. D. Shapiro, R. Bingham, J. M. Dawson, Z. Dobe, B. J. Kellett, and D. A. Mendis, J. Geophys. Res. 104, 2537-2554, doi: 10.1029/ 1998JA900047 (1999).

${ }^{7}$ B. F. McMillan and I. H. Cairns, Phys. Plasmas 14, 012103 (2007).
${ }^{8}$ I. H. Cairns and G. P. Zank, Geophys. Res. Lett. 29, 1143, doi: 10.1029/ 2001GL014112 (2002).

${ }^{9}$ G. Ganguli, L. Rudakov, W. Scales, J. Wang, and M. Mithaiwala, Phys. Plasmas 17, 052310 (2010).

${ }^{10}$ V. M. Kulygin, A. B. Mikhailovskii, and E. S. Tsapelein, Plasma Phys. 13, 1111 (1971).

${ }^{11} \mathrm{R}$. Davidson, Methods in Nonlinear Plasma Theory (Academic, New York, 1972).

${ }^{12}$ I. Roth, and M. Hudson, J. Geophys. Res. 88(A1), 483-488, doi:10.1029/ JA088iA01p00483 (1983).

${ }^{13}$ M. Mithaiwala, L. Rudakov, G. Ganguli, and C. Crabtree, Phys. Plasmas 18, 055710 (2011).

${ }^{14}$ K. G. McClements, R. Bingham, J. J. Su, J. M. Dawson, and D. S. Spicer, Astrophys. J. 409, 465 (1993).

${ }^{15}$ Yu. A. Omel'chenko, R. Z. Sagdeev, V. D. Shapiro, and V. I. Shevchenko, Sov. J. Plasma Phys. 15, 427-431 (1989).

${ }^{16}$ H. C. Koons and M. B. Pongratz, J. Geophys. Res. 86(A3), 1437-1446, doi:10.1029/JA086iA03p01437 (1981).

${ }^{17}$ G. Ganguli, L. Rudakov, M. Mithaiwala, and K. Papadopolous, J. Geophys. Res. 112, A06231, doi:10.1029/2006JA012162 (2007). 